\title{
Length distortion of temporally extended visual displays: Similarity to haptic spatial perception
}

\author{
J. D. BALAKRISHNAN, ROBERTA L. KLATZKY, and JACK M. LOOMIS \\ University of California, Santa Barbara, California \\ and \\ SUSAN J. LEDERMAN \\ Queen's University, Kingston, Ontario, Canada
}

\begin{abstract}
Three experiments were designed to investigate length distortion-the tendency to inflate estimates of the inferred distance between two points, as the length of a circuitous pathway between them increases. This phenomenon, previously demonstrated with haptic and locomotor exploration (Lederman, Klatzky, Collins, \& Wardell, 1987), was extended to vision through the presentation of pathways as a temporal sequence of illuminated points. The magnitude of the visual effect was less than that previously found in haptics, and conditions promoting the effect included a relatively slow presentation rate and moderately complex pathways. Commonalities in the pattern, if not the magnitude, of length distortion in vision and haptics suggest that similar processes of spatial encoding may underlie the phenomenon in both domains.
\end{abstract}

A pattern of systematic error, called length distortion, has been documented in haptic spatial perception (Lederman, Klatzky, \& Barber, 1985; Lederman, Klatzky, Collins, \& Wardell, 1987). It is a tendency to overestimate the inferred distance between two points, when a circuitous pathway is followed between them. The phenomenon has been found for both small-scale displays explored with the fingertip and large-scale displays explored through locomotion. In the present paper, we investigate whether a similar phenomenon occurs under certain conditions of visual exposure to a pathway.

The critical conditions are suggested by obvious differences between the circumstances under which spatial information is obtained in "movement space" (Lederman et al., 1987), as compared with visual perception. Specifically, haptic encoding is usually temporally extended; only a small, local sample of a spatial domain can be obtained at any one time.

Extending patterns over time imposes two kinds of demands: memory and integration. To consider the first of these, the loss of spatial information over time should profoundly impair the ability to construct a representation from a sequence of samples, in both haptics and vision. Short-term forgetting of movement and position information has been documented (as reviewed in Stelmach, 1982), as has the forgetting of the position of a dot in a visual display (Nelson \& Chaiklin, 1980; Taylor, 1961)

We acknowledge the support of the Office of Naval Research (contract to Roberta L. Klatzky and Susan J. Lederman). We also thank the UCSB Center for Spatial Cognition and Performance for access to multiuser equipment. Correspondence may be addressed to J. D. Balakrishnan, Department of Psychology, University of California, Santa Barbara, CA 93106. and in a haptic one (Lederman \& Taylor, 1969). The second (and related) demand imposed by temporally extended patterns is the need to integrate information, in order to obtain a global, survey type of spatial representation. Visual integration has been studied in experiments that involve an aperture- or slit-viewing procedure (Anstis \& Atkinson, 1967; Hochberg, 1968; Rock, 1981) or the presentation of spatially separated elements of a single form (Klatzky \& Thompson, 1975; Klopfer, 1985; Palmer, 1977; Thompson \& Klatzky, 1978). These studies indicate that integration over space and time is possible for visual displays, but within substantial constraints on viewing time and the particular form that is to be constructed. The importance of rate of exposure reinforces the assumption that temporal extension of patterns is likely to be a critical factor in the obtaining of visual length distortion.

There is reason to believe that length distortion would not occur if information about a static visual display were interrogated, reducing the memory/integration demands just described. In a relevant study, Thorndyke and HayesRoth (1982) found that people who learned about spatial layout from a map were unaffected by knowledge of the route between two points when estimating the interpoint direct distance. Thorndyke and Hayes-Roth suggested that distance estimation in this case was like an imaginal equivalent of tracing directly between the points on a map, a process that should not vary with a deviating route between them. The presence of length distortion suggests either that a "scannable" representation is not available, or that the representations of direct lengths in the internal images are systematically distorted by longer routes between endpoints.

Various aspects of the display and the manner of exploration affect the magnitude of haptic length distortion 
and might have similar effects in the visual equivalent. It has been found to increase with the length of the estimated distance and with the length of the pathway. Note that the pathway-length effect is not simply another version of the filled-space illusion (Krueger, 1970; Woodworth, 1938); subjects are judging not an indirect pathway itself, but the implicit distance between its endpoints. For small-scale pathways, distortion decreases with the rate at which the pathway is traversed. The effect is also quite robust: Error is reduced very little when the free hand is allowed to remain as an anchor at the starting point while the pathway is explored. This is somewhat surprising, because under these conditions, the explorer ultimately has to report only the distance between two statically maintained hands.

\section{EXPERIMENT 1}

Our purpose in this experiment was to determine whether length distortion could be found with temporally extended visual pathways, analogous to those used in previous haptics experiments. The stimuli were sequences of illuminated points following a path between two endpoints. The direct (endpoint-to-endpoint) length to be estimated, pathway length (in multiples of direct), and rate of pathway presentation were manipulated. If visual displays produce effects like those found with small-scale haptic patterns, length distortion should increase over all of these variables.

In addition, an anchor condition was simulated, in which the starting point remained illuminated as the pathway was traced. This condition allows for simultaneous presentation of the two points over which distance is to be estimated, even though a route is traced between them over time. If distributing information over time is critical to producing length distortion In vision, the simultaneous anchor might be expected to eliminate it. As noted above, however, a simultaneous haptic anchor has been found not to reduce length distortion substantially.

A final manipulation of the present displays was intended to affect the clarity of the pattern. In some trials, the position of points on the path was spatially perturbed, or jittered. This manipulation was based on known limits in spatial precision of the haptic perceptual system, which might produce uncertainty about the geometry of the pattern that is being traced. Despite its contribution to precise motor control, haptics appears to be intrinsically noisy in spatial localization, especially in comparison to vision. Both the cutaneous and kinesthetic systems that compose haptics can be evaluated in this regard. With respect to the cutaneous system, Loomis (1985) has modeled the fingertip as a bandwidth(resolution)-limited analogue of vision. Although the precision of the kinesthetic system is less well established, there is reason to believe that it is quite limited. Lederman, et al. (1985) asked subjects to indicate the distance and relative positions between the index fingers of the two hands. The fingers started out in a common location, and then one followed a straight path to some destination. For judgments of the resulting interdigit distance (Experiment 1), the errors were overestimations in the range of $50 \%-100 \%$ of the actual value $(2.5-15.2 \mathrm{~cm})$. Similar effects were found in a study (unreported) in which the fingers were statically anchored without movement between them. If overestimation errors in haptic length judgments reflect kinesthetic "noise"that is, positional uncertainty about points on the pathand if jittering imposes a parallel noise on the structure of the underlying pattern, it would be expected to increase length distortion. (The introduction of noise might be mediated by involuntary eye movements anticipating the local motion of points-see Kowler \& Steinman, 1981.)

\section{Method}

Subjects. Thirty subjects ( 18 females, 12 males) participated. In this and subsequent studies, the participants were students in introductory psychology who were partially satisfying a course requirement.

Apparatus and Stimuli. The participants were placed in a lightsealed chamber. A Conrac monitor (Model SNA 15/C) was placed at a distance so that the average length of the direct line between pathway endpoints subtended approximately $8.7^{\circ}$ of visual angle; viewing distance was not strictly controlled. The screen subtended $33.8^{\circ} \times 26.3^{\circ}$ of visual angle. A red acetate filter was placed over the monitor to reduce effective visual persistence. Under these viewing conditions, luminance was approximately $0.31 \mathrm{~cd} / \mathrm{m}^{2}$, as measured with a square test field that had the same intensity as the pathway points. There was virtually no visible trace of the path nor of the borders of the monitor.

The visual displays consisted of a sequence of illuminated points. A point was either 1 pixel in size or 3-the single pixel and the ones immediately above and to the right. With a probability of .05 , any point could be presented as a 3-pixel rather than a 1-pixel display (for reasons explained below). The distance between successive points was $0.27 \mathrm{~cm}$.

The set of stimulus pathways had three values of endpoint-toendpoint length: $1.6 \mathrm{~cm}\left(2.0^{\circ}\right), 7.0 \mathrm{~cm}\left(8.7^{\circ}\right)$, and $12.4 \mathrm{~cm}\left(15.4^{\circ}\right)$. The pathways were either the direct line between endpoints, or a zigzag line that made five angular changes between endpoints to form three peaks (see Figure 1). The pathway length for such paths was either three or five times the direct length. The specific pathways were formed starting with a path in which the three peaks were of equal height relative to the direct path and equally spaced, and then randomly perturbing the heights of the two "troughs" and

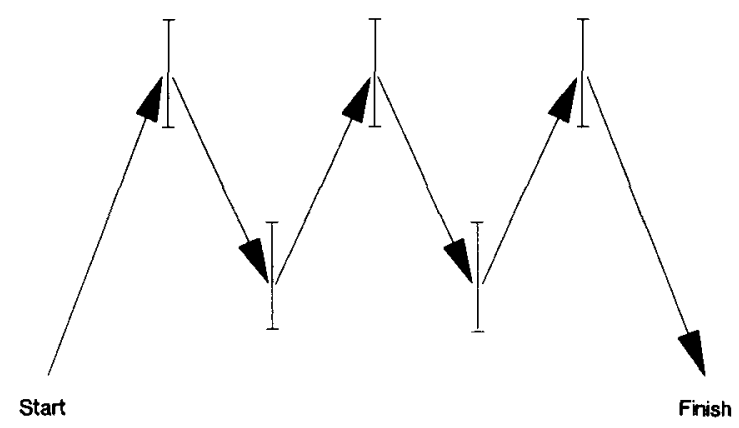

Figure 1. Method for deriving a typical pathway. Arrows represent pathway segments; vertical bars at angular changes represent variability in position of the change point. Height of rightmost peak is predetermined, given constraints of previous peaks and total pathway length. 
two (randomly chosen) peaks within $\pm 37.5 \%$. The remaining peak was then fully determined. The position of the pathway on the screen was randomly chosen as to orientation and absolute location, with some constraints. The longest pathways constrained the starting position, because starting points toward the center of the screen would have resulted in part of the pathway's lying off the screen. Thus longer paths started close to the perimeter of the screen and deviated inward. Pathways of other lengths were yoked to these same constraints on starting position.

The pathways were exposed through the illumination of each point in sequence at one of two speeds: fast $=1.8 \mathrm{~cm} / \mathrm{sec}$; slow $=$ $0.64 \mathrm{~cm} / \mathrm{sec}$. (These were selected on the basis of pilot data, as well as the subjective impression of fast or slow presentation. The fast rate is well below, and the slow rate somewhat below, the average speeds of $12.2 \mathrm{~cm} / \mathrm{sec}$ and $0.89 \mathrm{~cm} / \mathrm{sec}$ used for haptic exploration in Lederman et al., 1987.) An additional variable was whether the starting point of the path was left on as the other pathway points were illuminated (anchor condition), or whether only one point at a time was illuminated (no anchor).

As described above, an additional manipulation was intended to introduce noise about the structure of the pathway. Under one condition (no jitter), the originally designated pathway points were illuminated. Under the other (jitter), the illuminated points (other than the endpoints and peaks) were perturbed in a random direction. That is, the $x$ and $y$ coordinates were selected by adding, to the true value, a noise value that was sampled from a Gaussian noise distribution (mean $=0 ; S D=0.22 \mathrm{~cm}$; truncated at $\pm 4 S D$ ). There was still a subjective impression of an underlying smooth pathway.

With three levels of direct length, three of pathway length, and the anchor, speed, and jitter variables, there were 72 conditions. Each condition was presented on one trial, randomly ordered. Four practice trials, illustrating the various conditions, preceded the experiment proper.

Procedure. On each trial, the participant viewed a pathway. He or she was instructed to press the space bar as quickly as possible whenever a larger (i.e., 3-pixel) point was illuminated. This was a dummy task intended to encourage the participant to attend to the pathway, and performance was not monitored.

After the pathway terminated, a response display consisting of two points of light appeared on the lower portion of the screen. The participant was to move the rightward point by pressing the space bar, so as to adjust the distance between the points to match the apparent direct length. The initial interpoint distance was twice the direct length of the path. (This was done because the ballpark value of the target length was clearly apparent, and it would be frustrating to participants to adjust a long measurement when only a short path was to be estimated. Any systematic influence of initial position could be manifested in an effect of direct length but would not be expected to alter effects involving pathway length, because the starting point was constant over pathway lengths at each direct value.) The subject could either increase or decrease the distance within a single trial but not both, and was therefore instructed to avoid overshooting the target estimate. Reducing the distance was the default (correctly so), but the pressing of any other key prior to the space bar allowed the participant to increase the distance.

\section{Results}

The principal data consist of estimates in centimeters. The data are presented as a function of all of the principal variables in Figure 2. Analyses were conducted on the signed error data (estimate minus actual direct length), so that any effects of the direct-length variable do not simply reflect the fact that different lengths were being estimated. The main concern was with effects of the pathwaylength factor, which indicates the length-distortion effect, and its interaction with other variables.
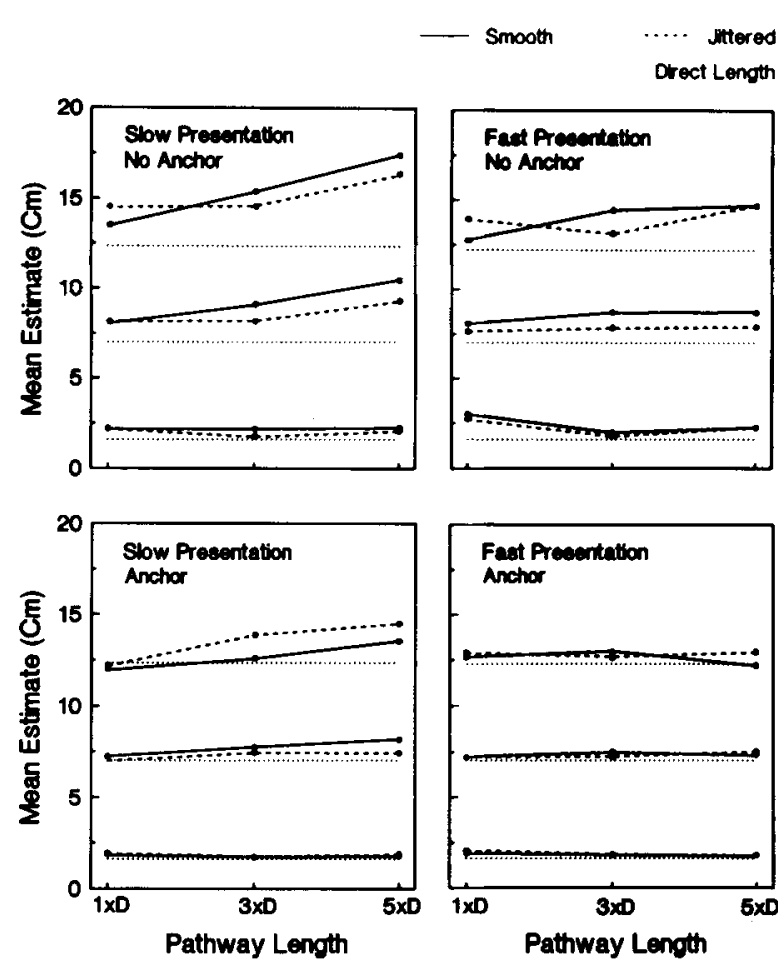

Figure 2. Mean estimates $(\mathrm{cm})$ in Experiment 1 by direct length (each group of functions is estimation for a different length), pathway length (in multiples of direct length, $D$ ), speed of pathway presentation, jitter versus smooth presentation, and anchor/noanchor.

A preliminary overall ANOVA on the errors revealed that all main effects except jitter were significant, that all two-way interactions except pathway-length $\times$ jitter, jitter $X$ speed, and anchor $\times$ speed were significant, and that the three-way pathway-length $x$ anchor $x$ jitter interaction was significant. The anchor clearly reduced overall error. Moreover, as the three-way interaction indicates, it changed the pathway-length and jitter effects. Thus, separate analyses were conducted for the anchor and noanchor conditions. Table 1 shows the $F$-values for those analyses.

When an anchor was present, there was still a small, significant effect of pathway length on errors, indicating length distortion. The effect increased with the estimated direct length. The average error was remarkably low for the shortest direct-length value. Although the pathwaylength $\times$ speed interaction was not significant, Figure 2 suggests that the distortion effect occurred primarily at the slow speed; the functions at the fast speed appear substantially flatter.

When there was no anchor, effects were generally larger and more complex. Length distortion was indicated by a significant effect of pathway length. The effect increased with the direct-length value and decreased with jitter and speed. The importance of the rate of presentation, as opposed to the duration of the entire presentation interval, is indicated by the fact that two conditions with virtually 
Table 1

Degrees of Freedom and $F$ Values in Experiment 1 for ANOVA on Signed Error $\times$ Anchor Present/No Anchor Present

\begin{tabular}{llcc}
\hline & & \multicolumn{2}{c}{$F$ ratio } \\
\cline { 3 - 4 } \multicolumn{1}{c}{ Source } & $d f$ & $\begin{array}{c}\text { No Anchor } \\
\text { Present }\end{array}$ & $\begin{array}{c}\text { Anchor } \\
\text { Present }\end{array}$ \\
\hline Direct length $(D)$ & 2,58 & $8.75 \ddagger$ & 0.99 \\
Pathway length $(P)$ & 2,58 & $9.32 \ddagger$ & $4.76^{*}$ \\
Speed $(S)$ & 1,29 & $6.92^{*}$ & 0.23 \\
Jitter $(J)$ & 1,29 & $7.10^{*}$ & 1.41 \\
$D \times P$ & 4,116 & $6.03 \ddagger$ & $4.73 \dagger$ \\
$D \times S$ & 2,58 & $6.29 \dagger$ & 2.42 \\
$D \times J$ & 2,58 & 1.69 & 0.89 \\
$P \times S$ & 2,58 & $5.39 \dagger$ & 0.40 \\
$P \times J$ & 2,58 & $6.77 \dagger$ & 0.04 \\
$S \times J$ & 1,29 & 0.54 & 0.00 \\
$D \times P \times S$ & 4,116 & 0.784 & 1.84 \\
$D \times P \times J$ & 4,116 & 1.58 & 0.67 \\
$D \times S \times J$ & 2,58 & 0.09 & 0.26 \\
$P \times S \times J$ & 2,58 & 0.54 & 1.01 \\
$D \times P \times S \times J$ & 4,116 & 0.25 & 0.12 \\
\hline${ }^{*} p<.05 . \quad \dagger<.01$. & $\ddagger p<.001$. & &
\end{tabular}

equal durations-12.4-cm direct, pathway $=5$, fast rate, versus $7-\mathrm{cm}$ direct, pathway $=3$, slow rate-showed very different degrees of distortion, with the smaller stimulus at the slower rate more prone to error.

To determine whether the pathway-length effect might in part reflect the number of responses made during pathway presentation, which would increase with its length, correlations were computed between the number of bright points (requiring space-bar responses during pathway presentation) and signed error, for each combination of pathway and direct length. These were uniformly small, ranging in absolute value from .02 to .11 , and none approached significance. This does not likely reflect small range; for example, in the lpngest pathway condition, the mean number of points was 11.6 with an $S D$ of 3.2 , but the correlation was only -.06 . Even had the correlations been significant, it is unclear why more responses would be expected to increase overestimation error in particular, as opposed to absolute error.

Another analysis was introduced because of the use of a horizontal response line. The well-known horizontal/ vertical illusion would suggest that relative to this line, vertical direct lines might be overestimated. (Note that this would not be expected to affect length distortion, because orientation was manipulated in the same way over all pathway lengths.) To address this possibility, we correlated the angular departure of the direct line orientation from the horizontal with the error, for each value of the direct-length and pathway-length factors. Several of these correlations were small but significant $(+.12$ to +.19 ), suggesting that the illusion might have been in effect. There was no tendency for larger correlations with longer pathways; in fact, the condition with maximum direct and pathway lengths had a nonsignificant correlation $(-.01)$.

\section{Discussion}

Experiment 1 demonstrates that length distortionincreased overestimation of straight-line distance due to the effects of an indirect pathway between points-can be found in vision, when the input is extended in time. As in pathways explored with the hand, the effect increased with slower presentation rates and with longer distances to be estimated. But unlike haptics, a simultaneous spatial anchor greatly reduced length distortion of visual patterns.

Although it is difficult to make direct comparisons across experiments in different modalities, the present effects appear to be less than those found previously with haptic displays. Previous haptic studies have found errors on the order of $100 \%$, given an increase in the pathway of five times the direct extent. Here, our maximum effect is on the order of $33 \%$ per fivefold increase. It could be argued that with slower speeds and longer direct-length values, higher errors could be obtained. However, the present speeds and values are within the range where haptic length distortion of small-scale displays is observed (Lederman et al., 1987).

The effect of jittering the pathway here was not as predicted. This manipulation was intended to introduce noise to the structure of the path rather than the startingpoint position. But oddly, jitter decreased length distortion (in the no-anchor condition; also note that the variances of the jitter and no-jitter errors were virtually identical). Since this does not replicate in Experiment 3 below, it will not be discussed further.

\section{EXPERIMENT 2}

In Experiment 1, the pathways were quite complex. Previous work on visual integration suggests that relatively simple configurations, or "good forms," are more likely to be integrated (Palmer, 1977; Thompson \& Klatzky, 1978). Conceivably, temporally extended pathways might be encoded into a representation approximating that of a simultaneous display, if they were simple enough. Following Thorndyke and Hayes-Roth (1982) and our suggestions in the introduction, no length distortion would then be expected, for subjects would simply ignore the pathway and scan mentally between the endpoints. Accordingly, in Experiment 2, we manipulated pathway complexity in terms of angular changes, independent of the length, presentation rate, and local features of the path (i.e., segments were straight lines or angles), to determine whether length distortion vanished with simple pathways.

Previous experiments on haptic length distortion have shown little evidence for such effects of pathway complexity, suggesting that an accurate survey representation is not obtained even for relatively simple patterns. The magnitude of the distortion effect was about as great for pathways that were straight lines with one angular change 
(Lederman et al., 1987) as for those that were complex curved contours (Lederman et al., 1985). It should be noted, though, that this comparison involves very different types of pathways in separate experiments.

\section{Method}

Subjects. Thirty students (17 females, 13 males) participated. All had normal vision, allowing correction. Three additional participants reported in a postexperimental interview that they were estimating pathway length rather than the implied direct length, and they were therefore eliminated from the analysis. (The estimation of pathway length was also quite apparent from the data.)

Stimuli and Design. The stimulus conditions and the general procedure were like those of Experiment 1, but with the following exceptions: A larger Conrac monitor (Model SNA 23/C), subtending $57.0^{\circ} \times 47.0^{\circ}$ of visual angle, was used. The distance between successive points was $0.51 \mathrm{~cm}$, and screen luminance was measured with the acetate filter at $0.65 \mathrm{~cd} / \mathrm{m}^{2}$. The two speeds were 2.43 and $0.85 \mathrm{~cm} / \mathrm{sec}$. There was no anchor condition, and no jitter. A complexity variable was introduced with three levels. The simplest pathways had one peak at the midpoint and thus formed an isosceles triangle. The other pathways had three or five peaks, the height and position of which were computed as in Experiment 1 . The tobe-estimated direct extents were $2.0 \mathrm{~cm}\left(2.5^{\circ}\right), 7.1 \mathrm{~cm}\left(8.8^{\circ}\right)$, and $12.2 \mathrm{~cm}\left(15.1^{\circ}\right)$. With three direct values, three pathway values (1, 3, or $5 \times$ direct length), three complexities, and two speeds, there were 54 conditions. Note, however, that when pathway = $1 \times$ direct length, the pathway is a straight line, and thus complexity becomes a dummy variable. Each condition occurred in one trial, randomly ordered. Practice trials occurred as before.

\section{Results}

The data are the direct-length estimates, averaged over subjects for each condition. Figure 3 shows these estimates. (Although there were distinct trials designated for the three complexities at pathway multiple $=1$, this is arbitrary, as was noted above, because the variable is a dummy. Hence the three sets of trials were simply aggregated and the points duplicated in the figure.)

Once again ANOVAs were conducted on the signed errors, with the principal interest in the pathway effect, in-

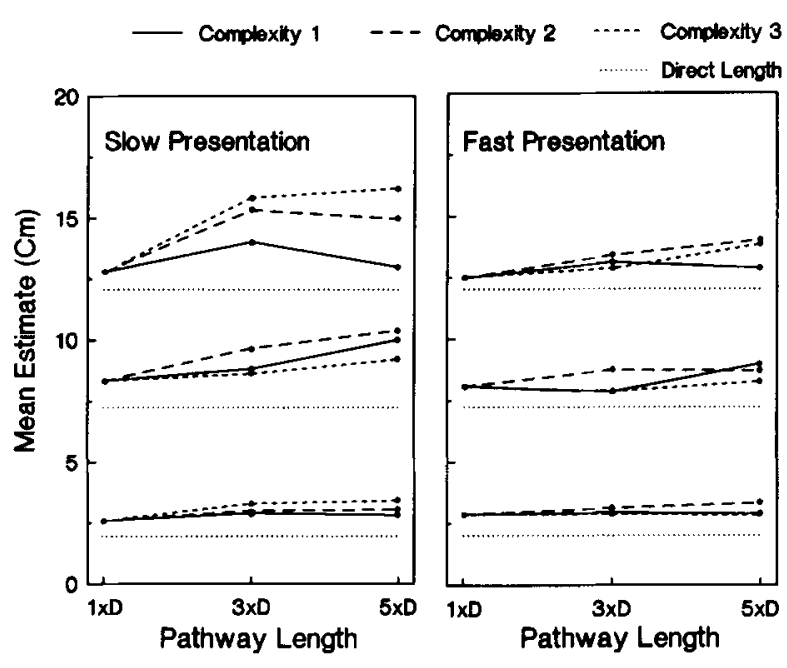

Figure 3. Mean estimates $(\mathrm{cm})$ in Experiment 2 by direct length (as in Figure 1), pathway length (in multiples of direct length, $D$ ), pathway speed, and stimulus complexity (1-3 angular peaks).
Table 2

Degrees of Freedom and $F$ Values in Experiment 2 for ANOVA on Signed Error

\begin{tabular}{llc}
\hline \multicolumn{1}{c}{ Source } & \multicolumn{1}{c}{$d f$} & $F$ ratio \\
\hline Direct length $(D)$ & 2,58 & $3.95^{*}$ \\
Pathway length $(P)$ & 2,58 & 2.35 \\
Complexity $(P)$ & 2,58 & $3.37^{*}$ \\
Speed $(S)$ & 1,29 & $19.79 \ddagger$ \\
$D \times P$ & 4,116 & 2.11 \\
$D \times C$ & 4,116 & $4.28 \dagger$ \\
$D \times S$ & 2,58 & $6.27 \dagger$ \\
$P \times C$ & 4,116 & 1.36 \\
$P \times S$ & 2,58 & $5.83 \dagger$ \\
$C \times S$ & 2,58 & 2.37 \\
$D \times P \times C$ & 8,232 & $2.16^{*}$ \\
$D \times P \times S$ & 4,116 & 1.94 \\
$D \times C \times S$ & 4,116 & 1.42 \\
$P \times C \times S$ & 4,116 & 0.58 \\
$D \times P \times C \times S$ & 8,232 & 0.48 \\
\hline
\end{tabular}

${ }^{*} p<.05 . \quad \dagger p<.01 . \quad \ddagger p<.001$.

dicating length distortion, and its interaction with complexity. Table 2 shows $F$ values in an overall ANOVA (entering the average value for pathway $=1$ at each level of complexity). This revealed a pathway-length $\times$ speed interaction, reflecting the finding of greater length distortion at the slow speed. When the fast-speed condition was considered alone, no effects were significant.

In addition, greater complexity caused error to increase, more so at longer direct-length values. The complexity effect is primarily between the least complex path-which forms an isosceles triangle-and the more complex ones. The latter do not differ significantly from one another. Most importantly, the three-way interaction among pathway length, direct length, and complexity was also significant, indicating the expected increase in length distortion with more complex pathways, at higher direct-length values.

Further analysis focused on the slow-speed trials, to investigate the pathway $\times$ complexity interaction. Pathwaylength effects were tested at each level of complexity, in an ANOVA on the pathway and direct-length factors. The pathway effect was significant only at the two higher complexity levels $[F \mathrm{~s}(2,58)=6.10$ and 4.17$]$ and interacted with direct length at the highest complexity $[F(4,116)=$ 3.87]. The absence of a pathway effect at the simplest complexity level does not mean that the task was error free (the variance for this condition was not particularly low, relative to the other complexity values), but that the trend toward systematically increasing overestimations (which can clearly be seen) was so small as to be nonsignificant. The effect at the two higher complexity levels reflects the shift from straight paths (pathway $=1 \times$ direct length) to angular ones (pathway $=3$ and $5 \times$ direct length); the latter two conditions did not differ significantly.

\section{Discussion}

Experiment 2 replicates the effects of the first: Pathwaybased distortion of direct-length estimates was found with temporally extended visual displays, especially at slow 
rates of presentation and longer direct extents. The maximal effect was to increase direct-length estimates by a bit more than $25 \%$, given a fivefold increase in pathway length.

In addition, this experiment shows that the complexity of a pathway increased error in estimation of the distance between its endpoints, more so as the to-be-estimated distance increased. Simple triangular pathways did not reveal substantial length distortion, whereas more complex ones did. However, the number of peaks in the more complex paths, beyond the simple triangle, did not have a significant effect. This contraindicates the use of a heuristic rule- "more peaks, longer end-to-end distance"- which should produce consistent increases in pathway estimates over increasing complexity (Sadella \& Staplin, 1980; Thorndyke, 1981).

\section{EXPERIMENT 3}

In Experiments 1 and 2, the subjects responded by adjusting an initial overestimation of the direct length, and adjustment was allowed only in one direction. Conceivably, this technique leads to systematic overestimation error, which might differ across the different values of direct length (although there is no obvious reason for pathwaylength effects within each direct-length value to be affected). Experiment 3 replicated Experiment 1, but with greater flexibility in response conditions.

\section{Method}

On each trial, the starting distance of the moveable point from the fixed endpoint on the response scale was randomly selected from an interval of $0.75 \times$ direct length to $1.25 \times$ direct length. Subjects could adjust the moveable point in either direction, using two texture-marked keys on the keyboard. The pressing of the space bar entered their response and gtarted the next trial. (Luminance was somewhat greater than in Experiment 1, at $0.86 \mathrm{~cd} / \mathrm{m}^{2}$.)

\section{Results}

Figure 4 shows the error for each anchor condition, each speed, and each combination of direct and pathway length. Because there was no effect of jitter within either anchor condition, the figure averages over the jitter and no-jitter conditions. Table 3 reports the $F$ values from ANOVAs on the anchor and no-anchor conditions.

Length distortion was observed in this study, although its magnitude was less than seen before. There were significant effects of pathway length in both conditions, as in Experiment 1. In the anchor condition, these effects were virtually zero at the two smaller values of direct length, producing an interaction. In the no-anchor condition, the pathway-length effect was again observed, and the interaction with direct length was significant, as in Experiment 1. However, unlike in that experiment, there was no significant interaction between pathway length and speed. Nevertheless, individual ANOVAs on each speed condition indicated that the pathway effect was significant only at the slow speed.

On the whole, the change in the response system appears to have had two effects: (1) elimination of the
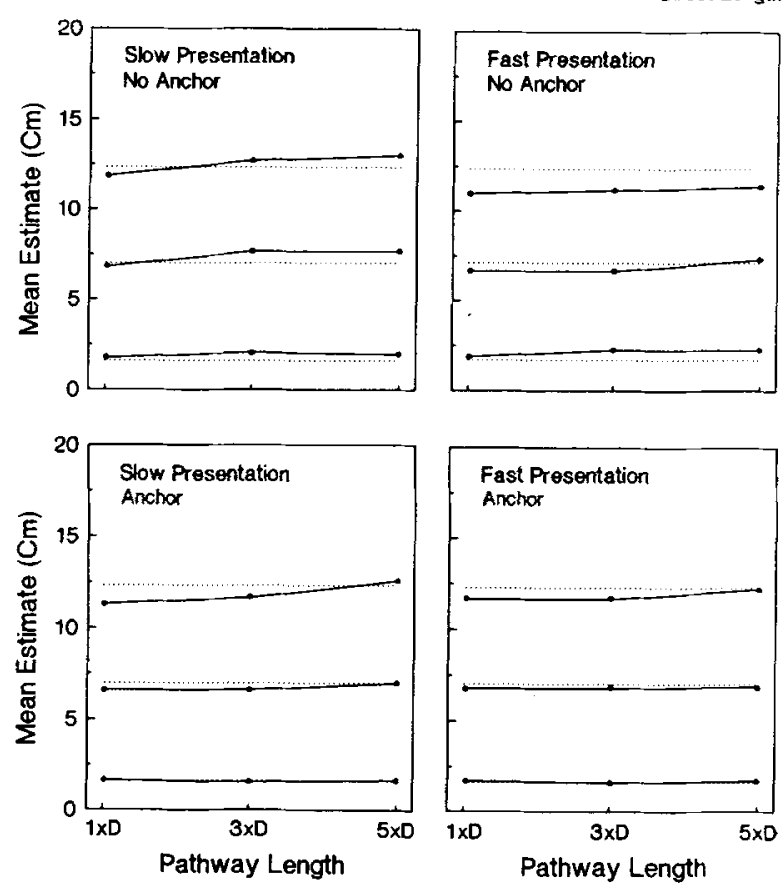

Figure 4. Mean estimates (cm) in Experiment 3 by direct length (as in Figure 1), pathway length (in multiples of direct length, $D$ ), speed of pathway presentation, and anchor/no-anchor.

general trend toward overestimation observed in the previous studies, and (2) reduction of the magnitude of length distortion (which here generally constitutes decreased underestimation of a direct route, the longer the indirect pathway between its endpoints). Nevertheless, the phenomenon was found in both the anchor and the noanchor conditions.

\section{GENERAL DISCUSSION}

The present experiments establish length distortionthe inflation of inferred direct length as pathway length increases-as a phenomenon that can be obtained in vision as well as touch. The conditions for producing a substantial effect appear to be temporally extended exposure, a moderately complex pathway, and a slow rate.

Table 3

Degrees of Freedom and $F$ Values in Experiment 3 for ANOVA on Signed Error $\times$ Anchor Present/No Anchor Present

\begin{tabular}{lccc}
\hline & & \multicolumn{2}{c}{$F$ ratio } \\
\cline { 3 - 4 } \multicolumn{1}{c}{ Source } & $d f$ & $\begin{array}{c}\text { No Anchor } \\
\text { Present }\end{array}$ & $\begin{array}{c}\text { Anchor } \\
\text { Present }\end{array}$ \\
\hline Direct length $(D)$ & 2,38 & $13.94 \ddagger$ & $10.57 \ddagger$ \\
Pathway length $(P)$ & 2,38 & $5.02^{*}$ & $7.45 \dagger$ \\
Speed $(S)$ & 1,19 & $13.33 \dagger$ & 0.01 \\
$D \times P$ & 4,76 & 0.91 & $8.7 \ddagger$ \\
$D \times S$ & 2,38 & $6.53 \dagger$ & 0.60 \\
$P \times S$ & 2,38 & 1.23 & 0.71 \\
$D \times P \times S$ & 4,76 & 1.41 & 0.28 \\
\hline${ }^{*} p<.05 . \quad \dagger p<.01$. & $\ddagger p<.001$. &
\end{tabular}


Both the present visual displays and patterns felt with the hand tend to produce an increase in distortion for longer direct lengths and slower rates of pathway traversal. However, there were also differences between the two situations: (1) The magnitude of the effects found here was generally less than those found in haptics, and overall accuracy was also much higher. (2) The effect has been found even with triangular (although not necessarily isosceles) pathways in haptics but was not found here with simple displays. (3) An endpoint anchor reduced the present length distortion more than in haptics. Presumably, under these circumstances, where the endpoints are displayed simultaneously, the probability of a direct scan increases, reducing the influence of the pathway in the making of the distance judgment.

These differences generally show haptics to be more vulnerable to distortion than vision. However, none of these effects dictates that the qualitative nature of spatial processing underlying distortion differs.

Following the model of Thorndyke and Hayes-Roth (1982), length distortion would not occur if observers could construct a survey representation that did not systematically distort direct pathways, and if they could scan over it to produce their estimates. With the present task, this appears essentially to be achieved with simple pathways, fast presentation rates, short direct lengths, or some combination of these circumstances.

Under other circumstances, however, length distortion occurs. Two general accounts of this error pattern can be proposed: (1) The representation of spatial layout is distorted, and scanning is accurate. In this case, the question arises as to the basis for the distortion. For instance, subjects may preserve the pathway's length but have a tendency to align its points by "flattening" or straightening, thus inflating the end-to-end distance. Related alignment tendencies have been observed in cognitive mapping studies (Tversky, 1981). (2) The representation might not be sufficient to allow scanning, and the direct length is estimated by some other means that introduces length distortion. Spatial extent might be estimated, within some degree of uncertainty, by memory for the startingpoint position, for example. If uncertainty increases with pathway length, and the presence of the detour biases subjects to respond toward the large end of the apparent range, length distortion would be observed.

At present, there is no compelling reason to choose between these two alternatives. However, introspective reports of subjects in the Lederman et al. (1987) study suggest the conscious impression of scanning a survey representation, which would favor the first alternative. On the other hand, the decrease in effects in Experiment 3 relative to Experiments 1 and 2 suggests that response processes may also play a role. In particular, starting the response with an overestimation may encourage bias toward the large end of the range of uncertainty about the true value.

One issue that bears consideration is whether temporal extension is the causal factor in length distortion, or whether encoding through movement is also critical.
There is some reason to believe that afference from eye muscles gives little information about position, in contrast to the kinesthetic system in general (see Clark \& Horch, 1987, for a review of kinesthesis). Brindley and Merton (1960; but cf. Skavenski, 1972) found that anesthetizing the conjunctival sacs and excluding visual clues left subjects unaware of eye deviations imposed or prevented by the experimenters. This casts some doubt on the role of movement (as opposed to time) as the critical factor. Also, the importance of presentation rate in other experiments on temporally extended visual patterns, some with apertures or slits (maintaining constant display position), supports the importance of time per se. However, it should also be noted that reafference from motor commands appears to provide information about eye movements, leaving open the possibility of a contribution of the movement system (Helmholtz, 1867/1962). Coren's (1986) work also demonstrates that even readiness for eye movements can have a substantial effect on visual perception, veridical or illusory. (For a review of the relation of eye movements to visual localization, see Matin, 1986.)

Modeling of spatial distortion may have substantial implications in the applied domain, such as the construction of tangible graphics displays for the blind. To the extent that vision and haptics employ common processes for representing layout in space, a visual display that mimics aspects of haptic presentation may be a useful tool. The present studies provide an initial step, by demonstrating that a spatial distortion previously observed in movement space can be extended to visual space with appropriate constraints on display presentation.

\section{REFERENCES}

ANStis, S. M., Atkinson, J. (1967). Distortions in moving figures viewed through a stationary slit. American Joumal of Psychology, 80, 572-585.

Brindley, G. S., \& Merton, P. A. (1960). The absence of position sense in the human eye. Journal of Physiology, 153, 127-130.

Clark, F. J., \& Horch, F. J. (1987). Kinesthesia. In K. R. Boff, L. Kaufman, \& J. P. Thomas (Eds.), Handbook of perception and human performance: Vol. 1. Sensory processes and perception (pp. 162). New York: Wiley.

Coren, S. (1986). An efferent component in the visual perception of direction and extent. Psychological Review, 93, 391-410.

HeLmholtz, H. von (1962). Treatise on physiological optics (Vol. 3; J. P. C. Southall, Ed. and Trans.). New York: Dover. (3rd German ed. published 1867)

Hochberg, J. (1968). In the mind's eye. In R. N. Haber (Ed.), Contemporary theory and research in visual perception (pp. 309-331). New York: Holt, Rinehart \& Winston.

KlatzKy, R. L., \& ThOMPSON, A. (1975). Integration of features in comparing multifeature stimuli. Perception \& Psychophysics, 18, 428-432.

KLOPFER, D. S. (1985). Constructing mental representations of objects from successive views. Journal of Experimental Psychology: Human Perception \& Performance, 11, 566-582.

Kowler, E., \& Steinman, R. M. (1981). The effect of expectations on slow oculomotor control: III. Guessing unpredictable target displacements. Vision Research, 21, 191-203.

KRUEGER, L. E. (1970). Apparent combined length of two-line and fourline sets. Perception \& Psychophysics, 8, 210-214.

Lederman, S. J., Klatzky, R., B Barber, P. (1985). Spatial- and 
movement-based heuristics for encoding pattern information through touch. Journal of Experimental Psychology: General, 114, 33-49.

Lederman, S. J., Klatzky, R. L., Collins, A., \& Wardell, J. (1987). Exploring environments by hand or foot: Time-based heuristics for encoding distance in movement space. Joumal of Experimental Psychology: Learning, Memory, \& Cognition, 13, 606-614.

Lederman, S. J., \& Taylor, M. M. (1969). Perception of interpolated position and orientation by vision and active touch. Perception \& Psychophysics, 6, 153-159.

Loomis, J. M. (1985, November). A model of character recognition and legibility. Paper presented at the annual meeting of the Psychonomic Society, Boston, MA.

MAtin, L. (1986). Visual localization and eye movements. In K. R. Boff, L. Kaufman, \& J. P. Thomas (Eds.), Handbook of perception and human performance: Vol. 1. Sensory processes and perception (pp. 20.1-20.45). New York: Wiley.

Nelson, T. O., Chaikuin, S. (1980). Immediate memory for spatial location. Journal of Experimental Psychology: Human Learning \& Memory, 6, 529-545.

PAlmer, S. E. (1977). Hierarchical structure in perceptual representation. Cognitive Psychology, 9, 441-474.

Rock, I. (1981). Anorthoscopic perception. Scientific American, 244, 145-153.

Sadalla, E., \& STaplin, L. (1980). The perception of traversed distance: Intersections. Environment \& Behavior, 12, 167-182.
SkAvenski, A. A. (1972). Inflow as a source of extraretinal eye position information. Vision Research, 12, 221-230.

Stelmach, G. E. (1982). Memory for movement with emphasis on short term aspects. In J. A. S. Kelso (Ed.), Human motor behavior: An introduction (pp. 117-142). Hillsdale, NJ: Erlbaum.

TAYLOR, M. M. (1961). Effect of anchoring and distance perception on the reproductions of forms. Perceptual \& Motor Skills, 12, 203-230.

Thompson, A., \& KLATZKY, R. L. (1978). Studies of visual synthesis: Integration of fragments into forms. Joumal of Experimental Psychology: Human Perception \& Performance, 4, 244-263.

ThORNDYKe, P. W. (1981). Distance estimation from cognitive maps. Cognitive Psychology, 13, 526-550.

ThORNDYKe, P. W., \& HAyes-Roth, B. (1982). Differences in spatial knowledge acquired from maps and navigation. Cognitive Psychology, 14, 560-589.

TVERSKY, B. (1981). Distortions in memory for maps. Cognitive Psychology, 13, 407-433.

WoOdWORTH, R. (1938). Experimental psychology. New York: Holt.

(Manuscript received September 1, 1988; revision accepted for publication March 8, 1989.) 